\title{
Humanoid co-workers: How is it like to work with a robot?
}

\author{
Ajay Vishwanath ${ }^{1}$, Aalind Singh ${ }^{2}$, Yi Han Victoria Chua ${ }^{3}$, Justin Dauwels ${ }^{3}$ and Nadia Magnenat-Thalmann ${ }^{4}$
}

\begin{abstract}
Human-robot interaction in corporate workplaces is a research area which remains unexplored. In this paper, we present the results and analysis of a social experiment we conducted by introducing a humanoid robot (Nadine) into a collaborative social workplace. The humanoid's primary task was to function as a receptionist and provide general assistance to the customers. Moreover, the employees who interacted with Nadine were given over a month to get used to her capabilities, after which, the feedback was collected from the staff on the grounds of influence on productivity, affect experienced during interaction and their views on social robots assisting with regular tasks. Our results show that the usage of social robots for assisting with normal day-to-day tasks is taken quite positively by the co-workers and that in the near future, more capable humanoid social robots can be used in workplaces for assisting with menial tasks. Finally, we posit that surveys such as ours could result in constructive opinions based on technological awareness, rather than opinions from media-driven fears about the threats of technology.
\end{abstract}

\section{INTRODUCTION}

Robots are becoming an inherent part of our daily lives [1][2]. Robots are not new, as historically, industrial robots [3] have been around since the 1970s. These industrial robots are bulky, sophisticated and powerful, as they are required to perform lifting, manipulating and various other mechanical tasks. Due to this powerful form factor, the practice has always been to ensure the maximum safety of people around these machines; therefore, there was little interaction between them. However, in the 2000s and later, friendlier and safer robots began to emerge, mainly service robots [4] and humanoids [5][6]. This increase could be attributed to the development of more sophisticated software and better electronic hardware to process information. Due to this ubiquitous influence in society, the study of humanrobot interaction (HRI) has become a necessity in this day and age.

On the acceptance of robots, there have been studies on larger groups about the impact of artificial intelligence (AI) and robotics on society and jobs [7][8][9]. These dystopian concerns have been the theme of recent years with futurists

1 Ajay Vishwanath is with Institute for Media Innovation, Nanyang Technological University, 50 Nanyang Drive, Singapore avishwanathentu.edu.sg

2 Aalind Singh is with Vellore Institute of Technology interning at Institute for Media Innovation, Nanyang Technological University, 50 Nanyang Drive, Singapore singh.aalind@gmail.com

3 Yi Han Victoria Chua and Justin Dauwels are with the School of Electrical and Electronic Engineering, Nanyang Technological University, 50 Nanyang Avenue, Singapore jdauwelsentu. edu.sg

4 Nadia Magnenat-Thalmann is with Institute for Media Innovation, Nanyang Technological University, 50 Nanyang Drive, Singapore and MIRALab, University of Geneva, Switzerland nadiathalmannentu.edu.sg such as Stephen Hawking and Elon Musk warning about the dangers of AI. While such an intelligent AI may exist far ahead in the future, we know for sure that more robots are featuring in our daily lives and it is important to understand whether people are ready for this change; there is a need to gauge our emotions to ensure the changes are not too abrupt.

To help understand this technological transformation, we present a preliminary case study where a humanoid robot, Nadine [6], works alongside customer service agents in an insurance company ${ }^{1}$. These service agents help customers to process change requests, explain company schemes, help sign up into new services and manage feedback. On the other hand, Nadine was assigned a portion of customer service tasks: answering customers' questions about products and services, assisting them with sign-ups to the company web's portal and being a conversational partner, entertaining the clients. We surveyed to capture the service agents' feelings and opinions about Nadine's presence in their service centre.

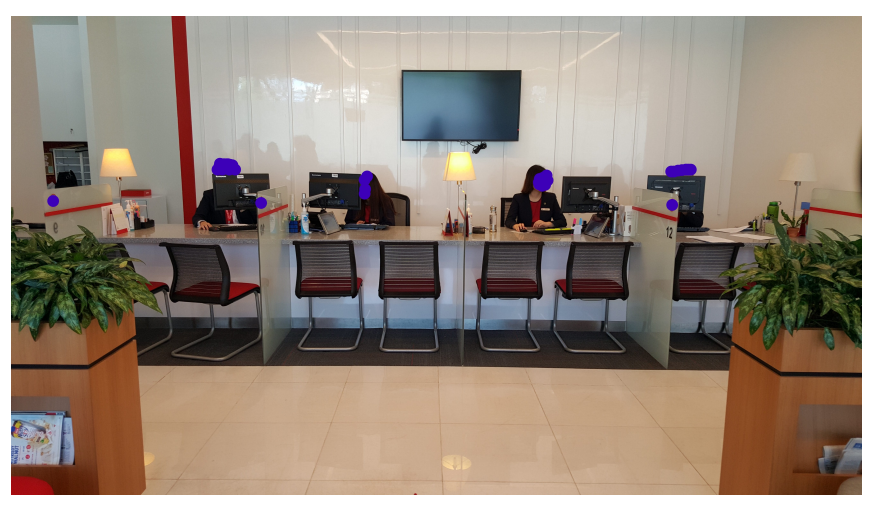

Fig. 1. Customer service agents at the insurance company.

Also, the humanoid was helping these customers to sign up to the company's web portal. Our research goal is to obtain feedback from the staff about their experience and make a comparison with other research studies. With these comparisons, we aim to continue a conversation on robots in workplaces. In the next section, we summarise related work in the field of human-robot interaction and public apprehensions about robots taking over jobs. In Section III and IV, we explain our experimental procedures and results, respectively, and we offer concluding remarks in Section V and VI.

\footnotetext{
${ }^{1}$ We will be maintaining the anonymity of the company throughout this article.
} 


\section{RELATED WORK}

Since HRI has been well explored in healthcare and social service workplaces [10], we extend this by focusing on corporate employees interacting with robot co-workers. Following this, we review reports about public acceptance of robots.

\section{A. Human-Robot Interaction in Corporate Workplaces}

A group of researchers [11] introduced a collaborative robot into three different manufacturing sites. The humanoid exhibited one human aspect, i.e., expression of emotions through a pair of eyes displayed on a monitor on the top of the robot. This experiment was an ethnographic study, where observations were made qualitatively. Seventeen participants across organisations highlighted the importance and suggested improvements in the sociality of the robot. While self-reported emotions from the employees were outside the scope of the study, the study provided useful guidelines in designing more social humanoid co-workers. A similar study [12] considered a telepresence robot in healthcare. While the staff welcomed the technology, they expressed concerns about privacy.

A study by Riether et al. [13], compared the effects of human versus robot presence on human task performance. Participants completed a battery of cognitive and motor tasks of varying difficulties alone, with a human confederate or an anthropomorphic robot head. The robot head, Flobi, consisted of exchangeable modular parts, e.g., facial features such as hairstyle, lips, eyebrows, carrying out one-on-one interaction with the human confederate. Riether and his colleagues found that working with a human or robot head had comparable social facilitation effects over working on tasks alone. These results suggest that a robopresence can be productive, but a major constraint in this study was the performance, as the robot was unable to change the facial modalities (eye movement, lip synchronisation, emotion generation) and had to be manually changed each time, thus making the interaction process robotic. In ours, we use a realistic humanoid, Nadine, which can autonomously mimic human facial modalities.

Another study conducted by Kim et al. [15] dwells more into the psychological domain of human-robot communication. They surveyed a total of 230 students on determining their concern for five communication constraints (feelings, non-imposition, disapproval, clarity, and effectiveness) in situations involving humans or robots. The results showed that people were more concerned about humans' feelings as compared to that of the humanoid counterpart. It was also observed that people were more drawn towards the social-oriented constraints while communicating with humans, which humanoid robots lack. This problem can be mitigated to a certain extent by incorporating emotions and other modalities into the humanoid robots as is done in the case of Nadine [16].

In 2009, a study [17] was conducted by Weiss et al. to measure the user experience (UX) factors in HRI, where the participants were asked to give voice commands to an HRP-2 robot, such as "pick up orange ball" and "turn to left". After the interaction, the participants answered questionnaires and provided a retrospective Think-out-loud about their experiences and opinions. Participants accepted the robot in terms of usability (SUS questionnaire) and the "Negative attitude toward situations of interactions with robots" variable showed a decrease before and after the experiment (NARS questionnaire). Also, half of the participants view the impact of robots negatively, and the rest view positively. A study with a higher sample space (greater than four participants) and linkage of UX to the impact of robots in society, could further this work.

\section{B. Public acceptance of robotics and automation}

Several works discussed here have tried to capture the general public's opinion on the rise of robots in society. Decker et al. [18] divided the market into various job segments based on the levels of difficulty and creativity required for performing the job. They further posit that machines might be more comfortable in doing routine jobs and might be better suited for certain difficult tasks such as deep ocean imaging compared to humans, but there are various areas where machines might not be able to match the thinking ability of humans. They also present how human-machine cooperation should be developed from a work science perspective and assert that robots should be viewed as more of an assistive technology rather than a substitution phenomenon.

Brougham et al. [7] conducted online and offline surveys in New Zealand to estimate the correlation of some social factors with Smart Technology, Artificial Intelligence, Robotics, and Algorithms (STARA) awareness. They found that greater STARA awareness was negatively related to organisational commitment and career satisfaction. The authors also note that most of the participants in this study were unaware of the advancements of these technologies; hence, they did not feel threatened by STARA's impact on job loss and careers.

On the other hand, an American survey [8] along similar lines found a link between the fear of advancements in robotics and AI, and, the threat of technological unemployment. They found that people with a high level of fear of technologies (called technophobes) are more likely to exhibit anxiety-related mental health issues. However, they have contradictory results to the former survey [7], as they cite that lack of technological awareness could give rise to these fears [8]. Similar studies conducted in Europe [9] [19] concluded that people's perception of robots has been growing more negative due to possible job losses.

In each of the above studies, the majority of the participants were not exposed to a fully autonomous humanoid robot. Therefore, their contradictory opinions could be a result of media coverage of technology and science fiction rather than informed decisions. In our study, we first introduce a social robot in the workplace before asking the company employees for their opinions. As we will see later, this approach could result in a more holistic idea about the robots and result in a richer survey response. 


\section{PROCEDURE AND SETUP}

This section details the humanoid's architecture and the alterations that we implemented to facilitate its integration into the workplace.

\section{A. Architecture}

Nadine is a socially intelligent humanoid robot, and its architecture is based on a typical Perception-Decision-Action model. The perception layer recognises various cues from the surrounding environment using modules such as face recognition, gesture recognition, 3D hand pose estimation [20], and, some intent identification from the social situations. With regards to decision, a behaviour tree planner is used to trigger the other sub-modules such as emotion and memory models, as well as social attention coupled with a dialogue manager. Finally, the action/interaction layer consists of a dedicated robot controller which includes emotion expression, lips synchronisation, hand gesture animation, and gaze generation.

Nadine has a realistic human appearance with natural skin and hair. The humanoid has 27 degrees of freedom, making her able to coordinate body movements effectively [21] [22]. Nadine can also recognise people that she has met before and engage in flowing conversations. She can also help people with reading text, show images, put on Skype sessions and respond to emails. Nadine can be seen as a part of human assistive technology [23], as she can assist people for continuous periods without any interruptions.

\section{B. Survey Setup}

In the service centre, Nadine [6] was seated separately from the human staff members. The main purpose of Nadine in the service centre was to answer Frequently Asked Questions (FAQs) about the service centre, insurance schemes, payment methods, etc. In addition, Nadine could look things up online, in case she could not find an apt result in the FAQ list. The setup is as shown in Figure 2 below.

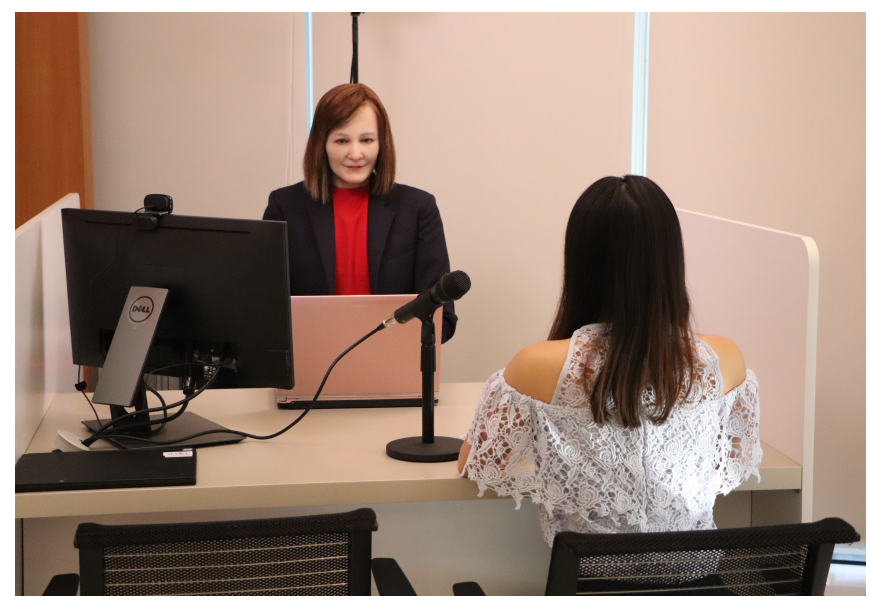

Fig. 2. Nadine working as a customer service agent (facing the camera).

For sure, the above-mentioned tasks could be achieved using a touchscreen computer or an ATM machine, but what makes Nadine special are the verbal (speech and language) and non-verbal (gaze, facial expression, gesture) behaviours. These capabilities are based on the perception-decisionaction loop, as mentioned in the subsection on Nadine's architecture. The inputs to Nadine are the captured through a microphone and Microsoft Kinect V2 sensor. These are then processed to recognise a person's speech, language, gestures, location and face, to name a few. Finally, this data is further processed to arrive at an appropriate decision. Therefore, in addition to the useful information, realistic face-to-face interaction is achieved.

Before Nadine was deployed in the field with customers, the agents interacted with her by: testing her abilities in FAQ answering, portal sign up and face recognition functionalities. During working hours, the staff went about their business as usual. Post-deployment and occasionally during lunch breaks or shift changes, they either spoke to Nadine or watched customers talking to the humanoid. The survey (see Table I) was conducted around a month after Nadine started doing her job.

\section{RESULTS}

Out of 13 respondents in the survey, 12 had interacted with Nadine. The survey was designed to capture different categories of co-worker appraisal: influence on own productivity, affect experienced during the interaction, and views on social robots in general.

\section{A. Productivity}

Nadine was trained to answer Frequently Asked Questions (FAQs) such as who is the CEO of the company?, where is the nearest bus stop? or How do you remit payment via internet banking?. These questions were based on the FAQs the company staff received from customers. When the company staff was asked whether their productivity was better, the same or worse, most of them felt that their productivity was the same as always or a little worse (Mean 2.15; SD 0.95). This could be attributed to the limited functionalities in the humanoid (See IVD-Miscellaneous Feedback).

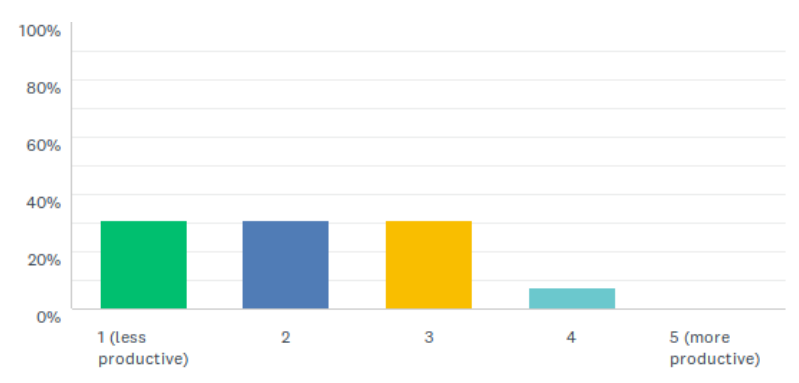

Fig. 3. Productivity.

\section{B. Positive and Negative Affect}

To capture the staff's affect while interacting with Nadine, we administered the I-PANAS-SF [24] questionnaire. We selected this questionnaire because it was on par with the original PANAS scale [25], and we could adhere to 
TABLE I

QUESTIONS AND OPTIONS PROVIDED DURING SURVEY

\begin{tabular}{|c|c|c|}
\hline \# & Questions & Options \\
\hline 1 & $\begin{array}{l}\text { Have you interacted with } \\
\text { Nadine? }\end{array}$ & Yes/No \\
\hline 2 & $\begin{array}{l}\text { Do you think Nadine is } \\
\text { adding value to the work- } \\
\text { force? }\end{array}$ & Yes/No \\
\hline 3 & $\begin{array}{l}\text { How much more produc- } \\
\text { tive are you because Na- } \\
\text { dine is answering Fre- } \\
\text { quently Asked Questions } \\
\text { (FAQ)? }\end{array}$ & $\begin{array}{l}\text { Scale of } 1 \text { (less productive) to } 5 \\
\text { (more productive) }\end{array}$ \\
\hline 4 & $\begin{array}{l}\text { How do you think Nadine } \\
\text { can improve? }\end{array}$ & $\begin{array}{l}\text { Choose one or more: } \\
\text { - Faster response and service } \\
\text { - More tasks and responsibility } \\
\text { - Customer/employee identifi- } \\
\text { cation } \\
\text { - Context awareness } \\
\text { - Friendliness } \\
\text { - Language understanding } \\
\text { - Other (please specify) }\end{array}$ \\
\hline 5 & $\begin{array}{l}\text { What do you think are } \\
\text { other uses of social robots } \\
\text { in the workplace? }\end{array}$ & $\begin{array}{l}\text { Choose one: } \\
\text { - Customer Support } \\
\text { - Receptionist } \\
\text { - Public Relations } \\
\text { - Not sure } \\
\text { - Other (please specify) }\end{array}$ \\
\hline 6 & $\begin{array}{l}\text { Which part of your tasks } \\
\text { would you delegate to a } \\
\text { social robot? }\end{array}$ & $\begin{array}{l}\text { Choose one: } \\
\text { - Handle customer support } \\
\text { emails } \\
\text { - Answering phone calls } \\
\text { - Customer feedback manage- } \\
\text { ment } \\
\text { - Handle Change Request } \\
\text { - Other (please specify) }\end{array}$ \\
\hline 7 & $\begin{array}{l}\text { Is XXXX brand image } \\
\text { improved by including } \\
\text { robots in the workplace? }\end{array}$ & Yes/No/Not sure \\
\hline 8 & $\begin{array}{l}\text { Would you like more so- } \\
\text { cial robots helping you } \\
\text { out? }\end{array}$ & $\begin{array}{l}\text { Choose one: } \\
\begin{array}{l}\text { - Yes } \\
\text { - No } \\
\text { - Maybe not now, but in the } \\
\text { future }\end{array}\end{array}$ \\
\hline 9 & $\begin{array}{l}\text { Thinking about yourself } \\
\text { and how you normally } \\
\text { feel while interacting with } \\
\text { Nadine, to what extent } \\
\text { do you generally feel: (I- } \\
\text { PANAS-X questionnaire) }\end{array}$ & $\begin{array}{l}\text { Alert, Inspired, Determined, Atten- } \\
\text { tive and Active, Upset, Hostile, } \\
\text { Ashamed, Nervous, Afraid (scale 1- } \\
\text { 5) }\end{array}$ \\
\hline 10 & $\begin{array}{l}\text { Any other sugges- } \\
\text { tions/comments? }\end{array}$ & Text field \\
\hline
\end{tabular}

the insurance company's request to keep the survey short. Positive affect feelings such as Alert, Inspired, Determined, Attentive and Active, and, Negative Affect feelings such as Upset, Hostile, Ashamed, Nervous and Afraid, are evaluated. Figure 4 and Table II show the statistics performed over the values obtained for these emotions.

For the above data, we perform a $t$-test with the following hypotheses:

$$
\begin{aligned}
& H_{0}: \mu_{p}=\mu_{n} \\
& H_{a}: \mu_{p}>\mu_{n}
\end{aligned}
$$

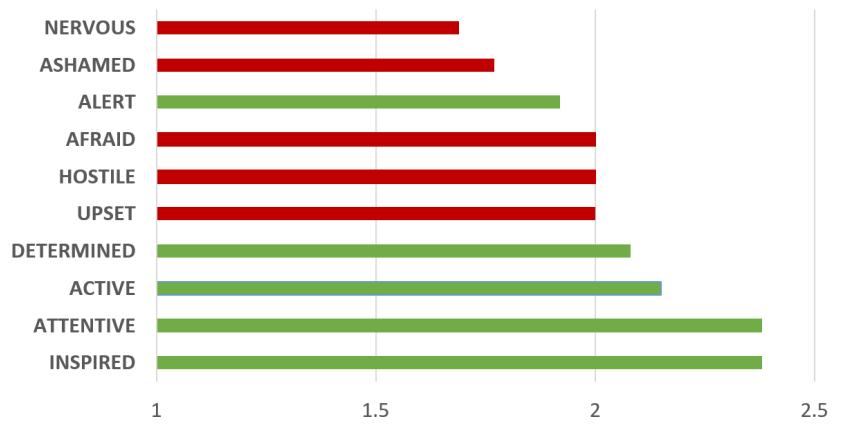

Fig. 4. I-PANAS-X emotion values on a scale of 5, in ascending order. Red and green bars signify negative and positive affect, respectively.

TABLE II

OVERALL POSITIVE AND NEGATIVE AFFECT VALUES OF THE STAFF.

\begin{tabular}{|l|l|l|l|l|}
\hline & Total & Count & Mean & Variance \\
\hline Positive Affect $(p)$ & 10.91 & 5 & 2.182 & 0.03962 \\
\hline Negative Affect $(n)$ & 9.46 & 5 & 1.892 & 0.02267 \\
\hline Difference $|p-n|$ & & & 0.29 & 0.01695 \\
\hline
\end{tabular}

where $H_{0}$ and $H_{a}$ are the null and alternate hypotheses, and, $\mu_{p}$ and $\mu_{n}$ are the means of positive and negative affect, respectively. Assuming statistical significance at $\alpha=0.05$, the one-tailed $t$-test results in a p-value of $0.04263\left(t_{\text {stat }}=\right.$ 2.132) which is lower than $\alpha$. Thus we can reject the null hypothesis.

Therefore, the overall affect experienced is not neutral but slightly tilted towards a positive notion. This suggests that the co-workers who interacted with Nadine may have experienced a positive affect.

\section{Views on Social Robots}

The participants of the survey were asked for their opinion on social robots like Nadine and social robots in general. The following was observed:

- When asked for areas of improvement of Nadine, 92\% felt that there could have been more tasks and responsibilities assigned to Nadine. This was followed by the need for response time, context awareness, and language understanding (see Fig. 5).

- Most of the respondents, $53.85 \%$ and $30.77 \%$, felt that social robots could be widely used as receptionists and customer support, respectively (see Fig. 6). The Others (Please specify) contained concierge.

- In terms of task delegation to social robots, Handling of Change Requests received the maximum votes (53.85\%). In other words, when a customer approaches a staff, changes in address, phone number or policy could be delegated to social robots (see Fig. 7). The Others (Please specify) contained answer customer simple request like change address or advice customer on the procedures and handle simple changes request and feedback management.

From the above responses, we can conclude that the service agents feel that robots can be of help with menial tasks 
such as handling change requests, receptionist duties, and, customer support. The fact that respondents feel that delegation and not replacement is critical, and will be helpful to introduce more robots into service centres. The employees understood Nadine's capabilities; hence they don't feel negatively towards Nadine perhaps because they don't think they can be substituted.

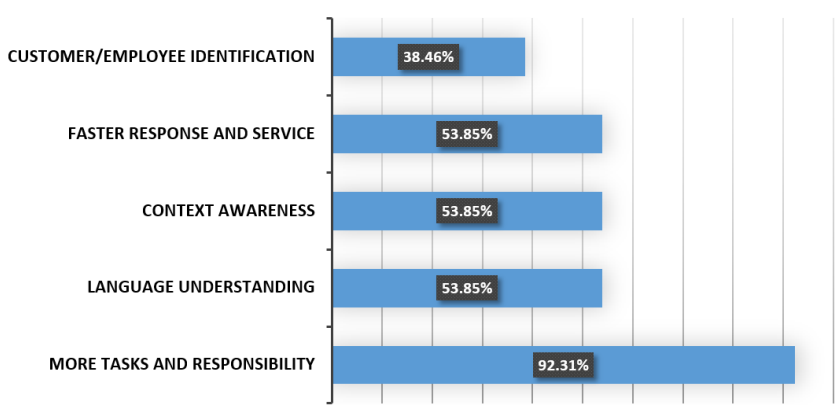

Fig. 5. Areas of improvement of Nadine.

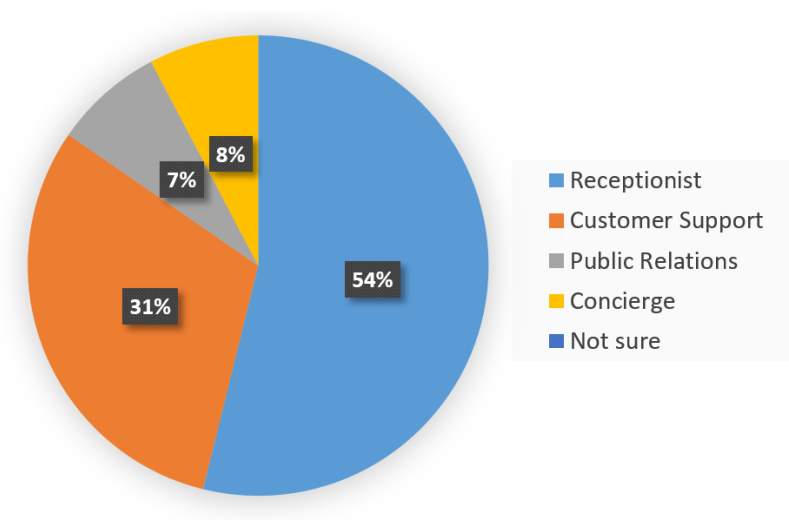

Fig. 6. Uses of social robots in general.

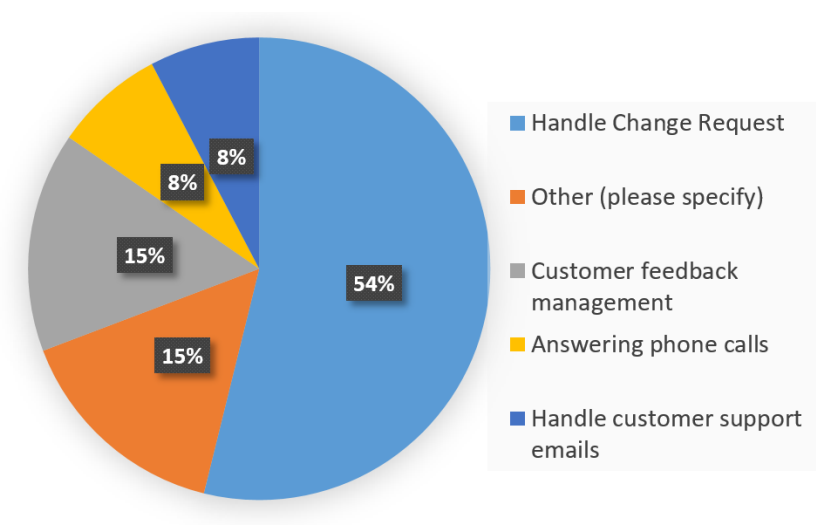

Fig. 7. Delegation of specific tasks to social robots.

\section{Miscellaneous Feedback}

From Table III, we can observe that the staff were impressed by her appearance, behaviour and communication capabilities. However, they felt that the capabilities (FAQ and registration process) were too limited to be of much help. This validates the claim in the previous result, where the staff voted for the assignment of more responsibilities and delegation of tasks.

\begin{tabular}{|c|}
\hline Comments by participants \\
\hline $\begin{array}{l}\text { nadine should have further responsibilities and answer ques- } \\
\text { tions which are more insurance related. }\end{array}$ \\
\hline $\begin{array}{l}\text { her hands are too big as commented by many customers. they } \\
\text { are, however, impressed that she looks like a real human. }\end{array}$ \\
\hline $\begin{array}{l}\text { would be good if nadine could complete a single task like } \\
\text { sign up XXXXX will be good as the robot have yet to help } \\
\text { in anyway. }\end{array}$ \\
\hline $\begin{array}{l}\text { if shes able to answer insurance related requests in XXX and } \\
\text { able to process simple request transactions, she will be more } \\
\text { useful in our workplace. }\end{array}$ \\
\hline
\end{tabular}

TABLE III

A LIST OF COMMENTS MADE BY THE STAFF DURING THE SURVEY.

\section{DISCUSSION}

There are several reports and studies on apprehensions of employees and their futurist views on robot coworkers [7] [8], and experiments in human-robot interaction in a factory setting [11]. Savela et al. [10], who conducted a systematic literature review about social acceptance of robots in different occupation fields concluded that, when participants of a study were exposed to robots, positive attitudes occurred more frequently. However, this conclusion mostly stems from surveys in the healthcare [26] [27] and social services sectors. To the best of our knowledge, there has been no survey conducted among employees after they have seen a real robot work in their insurance service centre. We can summarise our survey as follows:

- The productivity of the staff did not change due to the restricted number of functionalities of the humanoid.

- The staff exhibited an overall positive affect (Inspired, Active, Attentive) during their interaction with Nadine.

- After understanding the working of Nadine, they did not feel threatened about losing their jobs; instead, they highlighted that more social robots were needed to perform menial tasks in the service centre.

The analysis of the insurance service agents also hints at a similar [26] positive result. In general, employee exposure to robots leading to optimistic attitudes could be something to explore in future research across populations.

There are limitations in our work such as a small sample size $(N=13)$, restriction to a specific occupation-type, and the capturing of staff's affect in a specific instance of time. Despite these limitations, we outline some preliminary insights and realize our goal to further the conversation on robots in collaborative workplaces. Our study suggests that understanding the abilities of humanoid co-workers 
can facilitate acceptance of co-working with robots. We observe that negative feelings towards AI is perpetuated by catchy articles portraying AI and robotics as destructive to humanity [28]. We expect more studies on this topic in the future and encourage for practical HRI experiments over futurist surveys.

\section{CONCLUSION}

A humanoid robot, Nadine, was introduced in an insurance service center. After around one month, a survey was conducted to capture the feelings and opinions of the company staff. Overall, the staff may have had a positive outlook towards Nadine. While the staff's perceived productivity remained the same, they were optimistic about the potential roles accomplished by social robots. Also, a deeper analysis of the survey response conveys that they welcome more social robots to help with menial tasks such as handling change requests and customer support. Finally, there were no mentions of job loss due to automation. One could guess that the staff understood the limitations of AI and robotics to not feel threatened.

\section{ACKNOWLEDGMENTS}

This research is supported by the BeingTogether Centre, a collaboration between Nanyang Technological University (NTU) Singapore and University of North Carolina (UNC) at Chapel Hill. The BeingTogether Centre is supported by the National Research Foundation, Prime Ministers Office, Singapore under its International Research Centres in Singapore Funding Initiative.

Special thanks to our colleagues Manoj Ramanathan and Nidhi Mishra for their contribution to the experimental setup that laid the foundation for our study.

\section{REFERENCES}

[1] T. Sheridan, "Human-Robot Interaction", Human Factors: The Journal of the Human Factors and Ergonomics Society, vol. 58, no. 4, pp. 525532, 2016. Available: 10.1177/0018720816644364.

[2] A. Moniz and B. J. Krings, Robots Working with Humans or Humans Working with Robots? Searching for Social Dimensions in New Human-Robot Interaction in Industry, Societies, vol. 6, no. 3, p. 23, 2016.

[3] M. Rüßmann, M. Lorenz, P. Gerbert, M. Waldner, J. Justus, P. Engel, and M. Harnisch, "Industry 4.0: The future of productivity and growth in manufacturing industries.", Boston Consulting Group, 9(1), pp.5489, 2015.

[4] J. Forlizzi and C. Disalvo, Service robots in the domestic environment: a study of the roomba vacuum in the home, Proceeding of the 1st ACM SIGCHI/SIGART conference on Human-robot interaction - HRI 06, 2006.

[5] O. Mubin, C. J. Stevens, S. Shahid, A. A. Mahmud, and J. J. Dong, A Review Of The Applicability Of Robots In Education, Journal of Technology for Education and Learning, vol. 1, no. 1, 2013.

[6] J. Zhang, J. Zheng, and N. M. Thalmann, MCAEM: mixed-correlation analysis-based episodic memory for companionuser interactions, The Visual Computer, vol. 34, no. 6-8, pp. 11291141, 2018.

[7] D. Brougham and J. Haar, Smart Technology, Artificial Intelligence, Robotics, and Algorithms (STARA): Employees perceptions of our future workplace, Journal of Management \& Organization, vol. 24, no. 2, pp. 239257, 2017.

[8] P. K. Mcclure, Youre Fired, Says the Robot: The Rise of Automation in the Workplace, Technophobes, and Fears of Unemployment., Social Science Computer Review, vol. 36, no. 2, pp. 139156, 2017.
[9] T. Gnambs and M. Appel, Are robots becoming unpopular? Changes in attitudes towards autonomous robotic systems in Europe, Computers in Human Behavior, vol. 93, pp. 5361, 2019.

[10] N. Savela, T. Turja, and A. Oksanen, Social Acceptance of Robots in Different Occupational Fields: A Systematic Literature Review, International Journal of Social Robotics, vol. 10, no. 4, pp. 493502, 2017.

[11] A. Saupp and B. Mutlu, The Social Impact of a Robot Co-Worker in Industrial Settings, Proceedings of the 33rd Annual ACM Conference on Human Factors in Computing Systems - CHI 15, 2015.

[12] M. Niemel, L. V. Aerschot, A. Tammela, and I. Aaltonen, A Telepresence Robot in Residential Care: Family Increasingly Present, Personnel Worried About Privacy, Social Robotics Lecture Notes in Computer Science, pp. 8594, 2017.

[13] N. Riether, F. Hegel, B. Wrede, G. Horstmann: "Social facilitation with social robots?" HRI 2012: 41-48

[14] B. Kehoe, S. Patil, P. Abbeel, K. Goldberg, "A survey of research on cloud robotics and automation. IEEE Transactions on automation science and engineering", 12(2), pp.398-409, 2015.

[15] MS. Kim, J. Sur, and L. Gong, "Humans and humanoid social robots in communication contexts", AI and Society, 24:317, 2009

[16] N. Magnenat Thalmann, Nadine: A Human-like sociable and emotional Robot that remembers facts and people, 33rd Annual Conference in Computer Graphics International (CGI 2016), Heraklion, Crete, Greece

[17] A. Weiss, R. Bernhaupt, M. Tscheligi, E. Yoshida, "Addressing user experience and societal impact in a user study with a humanoid robot." In AISB2009: Proceedings of the Symposium on New Frontiers in Human-Robot Interaction (Edinburgh, 8-9 April 2009), SSAISB (pp. 150-157).

[18] M. Decker, M. Fischer, and I. Ott, Service Robotics and Human Labor: A first technology assessment of substitution and cooperation, Robotics and Autonomous Systems, vol. 87, pp. 348354, 2017.

[19] Special Eurobarometer 460, Attitudes towards the impact of digitisation and automation on daily life, May 2017

[20] L. H. Ge, H. Liang, J. S. Yuan and D. Thalmann, 3D Convolutional Neural Networks for Efficient and Robust Hand Pose Estimation from Single Depth Images, The 2017 IEEE Conference on Computer Vision and Pattern Recognition (CVPR 2017), Hawaii, USA, July 21-26, 2017

[21] A. Beck, Z. Zhang and N. Magnenat Thalmann, Motion Control for Social Behaviors, Context Aware Human-Robot and Human-Agent Interaction, Springer International Publishing, 237-256, 2015

[22] Y. Xiao, Z. Zhang, A. Beck, J. Yuan and D. Thalmann, HumanRobot Interaction by Understanding Upper Body Gestures, MIT Press Journals - Presence: Teleoperators and Virtual Environments, Vol. 23, No. 2, Pp. 133-154, 2014.

[23] N. Magnenat Thalmann, Z. Zhang, Social Robots and Virtual Humans as Assistive Tools for Improving Our Quality of Life, 5th International Conference on Digital Home (ICDH 2014), Guangzhou, China, November 28-30, 2014

[24] J. Karim, International Positive and Negative Affect Schedule ShortForm (I-Panas-SF): Testing for Factorial Invariance Across Cultures (June 23, 2011). Procedia Social and Behavioral Sciences, Vol. 15, pp. 2016-2022, 2011.

[25] J.R. Crawford, JD Henry, The Positive and Negative Affect Schedule (PANAS): Construct validity, measurement properties and normative data in a large nonclinical sample. British journal of clinical psychology. 43(3):245-65. Sep 2004.

[26] T. Turja, L. V. Aerschot, T. Srkikoski, and A. Oksanen, Finnish healthcare professionals attitudes towards robots: Reflections on a population sample, Nursing Open, vol. 5, no. 3, pp. 300309, 2018.

[27] Aaltonen, T. Turja, and M. Niemel, Comparison of Nursing Personnels User Experiences of Four Types of Assistive Robots: Challenges Include Knowledge and Safety Issues, Twelfth International Conference on Advances in Computer-Human Interactions Comparison, p. 147153, Feb. 2019.

[28] L. Floridi, "Robots, jobs, taxes, and responsibilities." Philosophy and Technology. 2017 Mar 1;30(1):1-4. 